\title{
Chemical Composition and Effects of Blended Essential Oil on Humans
}

\author{
Tapanee Hongratanaworakit*, Pongpat Srimuang, Ampot Wichanpreechar, Tharada Akarapattaranithi, \\ Benjaporn Taorang, Pimtida Rawdlaurh \\ Faculty of Pharmacy, Srinakharinwirot University, Thailand.
}

\section{ARTICLE INFO}

Article history:

Received on: 01/12/2016

Revised on: 18/12/2016

Accepted on: 30/12/2016

Available online: $31 / 01 / 2017$

Key words:

Aromatherapy, Mental

stimulation, Eucalyptus,

Rosemary, Pine, Patchouli.

\begin{abstract}
The blended essential oil was composed of eucalyptus, rosemary, patchouli, and pine oils. The blended oil was analyzed by GC-MS. The effects of blended oil on autonomic parameters and emotional changes in humans following inhalation were an investigation in this study. Thirty healthy volunteers participated in this study. Autonomic parameters, i.e. systolic blood pressure, diastolic blood pressure, mean arterial pressure and pulse rate were recorded. Additionally,emotional changes were assessed by means of visual analog scale. A quasiexperimental with one group pretest-post-test design was used to compare the differences in ANS parameters and emotional changes. Data was analyzed using paired t-test. The blended oil mainly contained 1,8-cineole (33.8\%), camphor (14.2\%), and $\alpha$-pinene (12.2\%). The blended oil showed significant increases in systolic blood pressure, diastolic blood pressure, mean arterial blood pressure and pulse rate which indicated an increase of autonomic arousal. In addition, inhalation of the blended oil led to activation at the behavioral level, i.e. subjects feel more alert, more vigorous, and more cheerful than before the administration of the oil. This finding points towards an increase of arousal in terms of self-evaluation. Thus, the effects of blended oil by inhalation may be characterized by the concept of stimulating/activating effects.
\end{abstract}

\section{INTRODUCTION}

Stress affects numerous body systems such as cardiovascular, respiratory, digestive, and immune systems (Cooksley, 1996; Damain and Damain, 1995). When stress occurs, some people often take medicines such as psychotropic drugs. If they use the medicines improperly, it will become addictive and harmful to the body.

Therefore the current trend of using natural products has gained in the field of alternative medicine. Essential oil, one of the natural products, is used to treat or alleviate the symptoms of both physical and mental disorders. The use of essential oils in psycho-aromatherapy is very popular. Some studies have shown that essential oils are used for mental strain or dullness and lethargy or exhaustion such as eucalyptus, rosemary, cajuput, pine, bergamot, patchouli, and marjoram oils

Corresponding Author

Tapanee Hongratanaworakit, Faculty of Pharmacy, Srinakharinwirot

University,Thailand.E-mail: tapanee @ g.swu.ac.th
(Albert and Steven, 1996; Lis-Balchin, 2006; Pujiarti et al., 2012). It is interesting that the main chemical components of these essential oils are 1,8-cineole, limonene, camphor, and $\alpha$-pinene which had been reported a stimulating effect (Heuberger et al., 2000; Kovar et al., 1987; Lis-Balchin, 2006; Steinmetz et al., 1987). Aromatherapy blending preparations may use alone or blend two, three or more oils together to match consumer's satisfaction. The blends usually smell better than a single oil. Blends of neroli and lavender showed an effective for postpartum mothers to improve physical and mental status (Imura et al., 2006). Blends of frankincense, bergamot, and lavender showed a positive effect on depression and pain on hospice patients with terminal cancer (Chang, 2008). Aromatherapy blending of rose, lavender, and sage oils was effective in decreasing the severity of menstrual cramps (Han et al., 2006). Rosemary oil exhibited stimulating effect, such as help to strengthen the brain, improve memory, fortify the heart, and energize mind (Atsumiand Tonosaki,2007; Diego et al., 1998; Hongratanaworakit, 2009; Lis-Balchin, 2006; Moss et al., 2003, Sayorwan et al., 2013). 
Rutledge and Jones (2007) recommend a combination of organically derived essential oils (eucalyptus, lemon, orange, camphor, rosemary, and peppermint) for fibromyalgia. Aromatherapists know that the refreshing effects of pine oil which communicate bracing, strengthening, and stabilizing psychological effects (Damain and Damain, 1995). Patchouli oil show stimulating effect on human physiological responses (Pujiarti et al., 2012).

Essential oils may be used alone or blend two, three or more oils together to match an individual's physical and emotional needs. Good blends usually combine all three notes. Top notes (such as eucalyptus, pine, and cajuput) are highly volatile and give the initial scent. Middle notes (such as neroli, rose, rosemary, and sweet marjoram) round out the blend and last a little longer. Base notes (such as patchouli, vetiver, and sandalwood) are long-lasting and acts as a fixative. Generally, the blends comprise of top note $20-40 \%$, middle note $50-70 \%$, and base note $10 \%$ (Hudson, 1999; Wildwood, 2000). In this study, we selected the essential oils that contained 1,8-cineole, limonene, camphor, and $\alpha$-pinene which had been reported a stimulating effect (Heuberger et al., 2000; Kovar et al., 1987; Lis-Balchin, 2006; Steinmetz et al., 1987). The eucalyptus (Eucalyptus globulus Labill.) and pine (Pinuspalustris Mill.) oils were used as a top note. The rosemary (Rosmarinus officinalis L.) oil was used as a middle note. The patchouli (Pogostemoncablin (Blanco) Benth.) oil was used as a base note.

There are a variety of approaches to evaluate the physiological and psychological effects of essential oil such as measuring changes in autonomic parameters, e.g. breathing rate, blood pressure, pulse rate, skin temperature and skin conductance, changes in brain wave activities, e.g. electroencephalogram, contingent negative variation, changes in mood, cognitive performances and emotion (Bensafi et al., 2000; Diego et al., 1998; Heuberger et al., 2000; Heuberger et al., 2006; Hongratanaworakit et al., 2005; Hongratanaworakit et al., 2007; Hongratanaworakit, 2009; Hongratanaworakit, 2010; Inoue et al., 2003; Moss et al., 2003; Rutledge and Jones, 2007). However, up to now, no experiments about the effects of blended oil on human autonomic parameters and on emotional changes after inhalation have been carried out. Therefore, the main objective of this study was: (a) to investigate the chemical composition of blended oil; (b) to evaluate the effects of blended oil on autonomic parameters, as well as on emotional changes in humans following inhalation.

\section{MATERIALS AND METHODS}

\section{Subjects}

Thirty healthy subjects (10 males and 20 females) aged between 21 and 23 years (mean age $22.27 \pm 2.67$ years) took part in the experiments. They were fully briefed, given written informed consent to all aspects of the study (Faculty of Pharmacy, Srinakharinwirot University Ethics Committees) and were free to withdraw at any time. Subjects did not show any symptoms of upper respiratory infections and did not have a history of cardiovascular disease. Forty-eight hours prior to testing subjects were asked to abstain from food, beverages, and toiletries containing the essential oil, as well as from any stimulants (for example, caffeine and nicotine).

\section{Essential oil preparation and administration}

The essential oils were obtained from Thai-China Flavours and Fragrances Industry Co., Ltd, Thailand. The blended oil was prepared in a volume ratio and composed of eucalyptus: rosemary: patchouli: pine oils $(2: 6: 1: 1)$. The blended oil was analyzed by GC/MS. Blended oil was diluted to $10 \% \mathrm{v} / \mathrm{v}$ concentration in swe et almond oil.

\section{Gas chromatography/mass spectrometry (GC/MS) analysis}

Chemical analysis of single/pure essential oils and blended oil was achieved by gas chromatography/mass spectrometry. GC/MS was carried out on a Trace GC Ultra/DSQ Quadruple spectrometer/Finnigan gas chromatograph fitted with a fused-silica BPX5 capillary column $(30 \mathrm{~m}, 0.25 \mathrm{~mm}, 0.25 \mu \mathrm{m}$ film thickness). The carrier gas was helium at $1.0 \mathrm{~mL} / \mathrm{min}$. Injector temperature was $180^{\circ} \mathrm{C}$. A split ratio of $1: 100$ was used. Transfer line temperature was $240^{\circ} \mathrm{C}$. Column temperature was $60^{\circ} \mathrm{C}$ for 1 min, then programmed from $60-240^{\circ} \mathrm{C}$ at $3^{\circ} \mathrm{C} / \mathrm{min}$. The MS were obtained from using an ionization energy of $70 \mathrm{eV}$ and ion source temperature of $200{ }^{\circ} \mathrm{C}$. The individual components were identified: (a) by comparing the Kovats gas chromatographic retention indices of the peaks on the HP-5MS column with the Adams Essential Oil Mass Spectra library; (b) by comparison of their MS with those stored in the MS database (NIST05 library) and with those reported in the literature (Adams, 2004; Lakusic et. al., 2013).

\section{Acquisition of autonomic parameters and emotional changes}

Autonomic parameters, i.e. systolic blood pressure (SBP), diastolic blood pressure (DBP), MAP (mean arterial blood pressure), and pulse rate (PR), were recorded simultaneously. The autonomic parameters were measured in the dominant arm by sphygmomanometer using an automated system (Digital Electronic Model DS-155E, Japan). Visual analog scales (VAS) were used to assess subjective emotional conditions. They consisted of $100 \mathrm{~mm}$ lines for 4 items: relaxation, vigor, mood and alertness. Each subject was asked to mark his or her feeling for each item between the two possible extremes: relaxed (on the left) and tense (on the right) for the item 'relaxation', vigorous (on the left) and feeble (on the right) for the item 'vigor', cheerful (on the left) and bad tempered (on the right) for the item 'mood', alert (on the left) and tired (on the right) for the item 'alertness' (Heuberger et al., 2006; Hongratanaworakit et al., 2005; Hongratanaworakit et al., 2007; Hongratanaworakit, 2009; Hongratanaworakit, 2010; Sayorwan et al., 2013).

\section{Experimental protocol}

The experimental protocol was modified from Diego et al. (1998). The pilot study to test the validity of the modified protocol was done in thirty healthy volunteers which were a different group from the experimental group. Lavender oil 
(considered a relaxing oil) and rosemary oil (considered a stimulating oil) were selected in the pilot study. In addition, the experimental protocol has been previously used by our group (Taorang et al., 2009). A quasi-experimental with one group pretest-post-test design was used to compare the differences in ANS parameters and emotional changes. Experiments were performed between 08.00 and $12.00 \mathrm{AM}$ to decrease circadian variation of ANS parameters. All experiments were conducted in a bright and quiet room. Ambient temperature was $25 \pm 1^{\circ} \mathrm{C}$. Upon arrival, the subjects were interviewed about their personal data, e.g. sex, age, height, weight. In addition, they were asked about the rating of emotional responses. After completion of the interview and the rating scales, autonomic parameters were measured. Blended oil was presented in $50 \mathrm{~mL}$ amber glass vial which the subjects held about 3 inches from their nose and inhaled continually for a period of six minutes. After completion of the trial, autonomic parameters were repeatedly measured at the end of the trial. The subjects were asked to rate the VAS.

\section{Statistical analyses}

All data are presented as the mean \pm standard error mean. The SPSS version 11.5 was used for statistical analysis. The effects of blended oil, lavender oil, and rosemary oil on ANS parameters and emotional responses were determined by comparing the mean values between before and after inhalation using paired t-test. A p-value $<0.05$ was considered statistically significant.

\section{RESULTS AND DISCUSSION}

In the present investigation blended oil was administered by sniff to healthy subjects. Autonomic parameters, i.e. SBP, DBP, MAP, and PR, were recorded as indicators of the arousal level of the autonomic nervous system (ANS). In addition, subjects had to rate their emotional condition in terms of alertness, vigor, and mood in order to assess subjective behavioral arousal. A quasiexperimental with one group pretest-post-test design was used to compare the differences in ANS parameters and emotional changes.

The pilot study to test the validity of protocol was done in thirty healthy volunteers which were a different group from the experimental group. Lavender oil (considered a relaxing oil) was selected in the pilot study. The mean and SEM of autonomic and emotional parameters of the lavender oil were presented in Table 1. Pre-post t-tests revealed the following significant autonomic changes. SBP, DBP, and MAP of subjects significantly decreased after sniffing the lavender oil $(\mathrm{P}<0.05$ for all). No significant effects of the lavender on PR was found ( $\mathrm{P}>0.05)$. In addition, subjects felt more relaxed and less alert than before the administration of the lavender oil $(\mathrm{P}<0.05$ for all). Inhalation of lavender oil decreased the level of arousal of the ANS, i.e. decreases of SBP, DBP, and MAP. Moreover, inhalation of lavender oil led to deactivation at the behavioral level, i.e. subjects feel more relaxed and less alert than before the administration of the oil. This is in accordance to a study done by Moss et al. (2003) and Sayorwan et al. (2012). Thus, the effects of lavender oil by inhalation may be characterized by the concept of relaxing effects.

Table 1: Mean and SEM of autonomic and emotional parameters for the lavender oil.

\begin{tabular}{cccccc}
\hline Parameters & PRE & POST & $\begin{array}{c}\Delta \text { Pre }- \\
\text { Post }\end{array}$ & $\boldsymbol{t}$ & $\begin{array}{c}\text { p } \\
\text { value }\end{array}$ \\
\hline SBP & $111.7 \pm 1.9$ & $107.8 \pm 1.6$ & +3.9 & +4.92 & $0.000^{*}$ \\
DBP & $74.4 \pm 1.0$ & $71.9 \pm 1.1$ & +2.5 & +3.14 & $0.004^{*}$ \\
MAP & $86.9 \pm 1.2$ & $83.8 \pm 1.1$ & +3.1 & +5.65 & $0.000^{*}$ \\
PR & $73.4 \pm 2.1$ & $72.2 \pm 1.9$ & +1.2 & +1.00 & 0.324 \\
RL & $46.1 \pm 3.4$ & $33.7 \pm 3.1$ & +12.4 & +3.59 & $0.001^{*}$ \\
AL & $45.6 \pm 3.6$ & $54.9 \pm 3.5$ & -9.3 & -2.88 & $0.007^{*}$ \\
\hline
\end{tabular}

SBP: systolic blood pressure DBP: diastolic blood pressure, MAP: mean arterial blood pressure, PR: pulse rate, RL: relaxation, AL: alertness, SEM: standard error mean. * Significant differences $(\mathrm{p}<0.05)$.

In addition, rosemary oil (considered a stimulating oil) was also selected in the pilot study. The mean and SEM of autonomic and emotional parameters for the rosemary oil were presented in Table 2. Pre-post t-tests revealed the following significant autonomic and emotional changes. SBP and PR of subjects significantly increased after sniffing the rosemary oil ( $\mathrm{P}<0.05$ for all). No significant effects of the rosemary on DBP and MAP were found ( $\mathrm{P}>0.05$ for all). Moreover, subjects felt more alert than before the administration of the rosemary oil $(\mathrm{P}<0.05)$. No significant effects of the rosemary oil on relaxation was found $(\mathrm{P}>0.05)$. Inhalation of rosemary oil increased the level of arousal of the ANS, i.e. increases of SBP and PR. Moreover, inhalation of the rosemary oil led to activation at the behavioral level, i.e. subjects feel more alert than before the administration of the oil. Our findings are consistent with the study done by Moss et al. (2003) and Sayorwan et al. (2013). Thus, the effects of rosemary oil by inhalation may be characterized by the concept of stimulating/activating effects.

From the pilot study showed that the protocol was accurate and can be used to test the blended oil.

Table 2: Mean and SEM of autonomic and emotional parameters for the rosemary oil.

\begin{tabular}{cccccc}
\hline Parameters & PRE & POST & $\begin{array}{c}\Delta \text { Pre - } \\
\text { Post }\end{array}$ & $\boldsymbol{t}$ & p value \\
\hline SBP & $111.4 \pm 1.8$ & $112.9 \pm 1.9$ & -1.5 & -2.20 & $0.028^{*}$ \\
DBP & $74.9 \pm 1.3$ & $75.3 \pm 1.4$ & -0.4 & -0.26 & 0.800 \\
MAP & $87.1 \pm 1.3$ & $87.8 \pm 1.3$ & -0.7 & -1.25 & 0.213 \\
PR & $73.6 \pm 2.0$ & $76.7 \pm 2.0$ & -3.1 & -3.21 & $0.001^{*}$ \\
RL & $46.2 \pm 2.7$ & $40.1 \pm 3.1$ & +6.1 & -2.25 & $0.024^{*}$ \\
AL & $44.4 \pm 3.6$ & $36.6 \pm 3.5$ & +7.8 & -2.00 & $0.045^{*}$ \\
\hline
\end{tabular}

SBP: systolic blood pressure DBP: diastolic blood pressure, MAP: mean arterial blood pressure, PR: pulse rate, RL: relaxation, AL: alertness, SEM: standard error mean. * Significant differences $(\mathrm{p}<0.05)$.

The mean and SEM of autonomic and emotional parameters for the blended oil are presented in Table 3. Pre-post ttests revealed the following significant autonomic and emotional changes. SBP, DBP, MAP, and PR of subjects significantly increased after sniffing the blended oil $(\mathrm{P}<0.05$ for all). In addition, subjects felt more alert, more vigorous, and more 
cheerful than before the administration of the blended oil $(\mathrm{P}<0.05$ for all).

Table 3: Mean and SEM of autonomic and emotional parameters for the blended oil.

\begin{tabular}{cccccc}
\hline Parameters & PRE & POST & $\begin{array}{c}\Delta \text { Pre - } \\
\text { Post }\end{array}$ & $\boldsymbol{t}$ & p value \\
\hline SBP & $111.2 \pm 1.9$ & $117.4 \pm 1.8$ & -6.2 & -8.67 & $0.000^{*}$ \\
DBP & $73.1 \pm 1.5$ & $75.6 \pm 1.2$ & -2.5 & -3.11 & $0.004^{*}$ \\
MAP & $85.8 \pm 1.5$ & $89.6 \pm 1.2$ & -3.8 & -6.04 & $0.000^{*}$ \\
PR & $74.6 \pm 1.7$ & $79.7 \pm 1.8$ & -5.1 & -5.38 & $0.000^{*}$ \\
AL & $44.7 \pm 3.1$ & $36.3 \pm 2.6$ & +8.7 & +2.83 & $0.009^{*}$ \\
V & $48.9 \pm 3.7$ & $41.2 \pm 2.6$ & +7.7 & +2.33 & $0.027^{*}$ \\
M & $35.9 \pm 3.1$ & $29.4 \pm 2.2$ & +6.5 & +2.63 & $0.014^{*}$ \\
\hline
\end{tabular}

SBP: systolic blood pressure DBP: diastolic blood pressure, MAP: mean arterial blood pressure, PR: pulse rate, AL: alertness, V: vigor, M: mood, SEM: standard error mean. * Significant differences $(\mathrm{p}<0.05)$.

The chemical composition of the single/pure essential oils and blended oil was determined by gas chromatography-mass spectrometry (GC/MS), as shown in Table 4. The blended oil mainly contained 1,8 -cineole $(33.8 \%)$, camphor $(14.2 \%)$, and $\alpha$ pinene $(12.2 \%)$. Limonene (3.7\%), $\beta$-pinene $(3.4 \%)$, and p-cymene $(3.5 \%)$ were the minor components found in the blend. The major component observed in the single/pure essential oils, eucalyptus and rosemary oils was 1,8-cineole (23.2-83.3\%), whereas the major components of pine oil were $\alpha$-pinene $(17.2 \%)$, terpinolene (15.1\%), and $\alpha$-terpinene (14.0\%).

Table 4: Chemical compositions of blended essential oil preparations and single essential oils.

\begin{tabular}{|c|c|c|c|c|c|c|}
\hline \multirow[b]{2}{*}{ Compounds } & \multirow[b]{2}{*}{$\mathbf{K I}^{*}$} & \multicolumn{5}{|c|}{ \%Area } \\
\hline & & 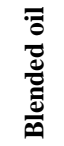 & 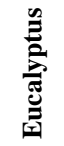 & 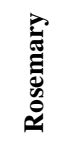 & 氞 & $\stackrel{\mathscr{E}}{\stackrel{\Xi}{E}}$ \\
\hline Tricyclene & 926 & 0.4 & - & 1.0 & - & 0.3 \\
\hline$\alpha$-Pinene & 939 & 12.2 & 6.8 & 19.2 & - & 17.2 \\
\hline Camphene & 954 & 6.8 & - & 13.2 & - & 4.1 \\
\hline$\beta$-Pinene & 979 & 3.4 & 1.1 & 6.8 & - & 0.1 \\
\hline Myrcene & 988 & 0.7 & 0.4 & 1.1 & - & 0.4 \\
\hline$\alpha$-Phellandrene & 1002 & - & - & 0.1 & - & 0.4 \\
\hline$\delta$-3-Carene & 1011 & - & - & - & - & 0.5 \\
\hline 1,4-Cineole & 1014 & 1.8 & - & - & - & - \\
\hline$\alpha$-Terpinene & 1017 & - & - & - & - & 14.0 \\
\hline p-Cymene & 1026 & 3.5 & 3.8 & 4.1 & - & 3.2 \\
\hline Limonene & 1029 & 3.7 & 4.6 & 3.5 & - & 8.8 \\
\hline 1,8-Cineole & 1031 & 33.8 & 83.3 & 23.2 & 0.1 & 7.8 \\
\hline$\gamma$-Terpinene & 1059 & 0.2 & - & - & - & 1.9 \\
\hline Terpinolene & 1088 & 1.4 & - & - & - & 15.1 \\
\hline Linalool & 1096 & 0.4 & - & 0.5 & - & - \\
\hline endo-Fenchol & 1116 & 0.4 & - & - & - & 2.2 \\
\hline$\alpha$-Campholenal & 1126 & - & - & 0.5 & - & - \\
\hline 1-Terpineol & 1133 & 0.8 & - & - & - & 4.0 \\
\hline cis- $\beta$-Terpineol & 1144 & - & - & - & - & 2.4 \\
\hline Camphor & 1146 & 14.2 & - & 19.2 & - & - \\
\hline Camphene hydrate & 1149 & - & - & - & - & 0.2 \\
\hline Isoborneol & 1160 & 1.2 & - & 1.3 & - & 0.6 \\
\hline Borneol & 1169 & 2.3 & - & 2.2 & - & 1.9 \\
\hline Terpinen-4-ol & 1177 & 0.2 & - & - & - & 0.6 \\
\hline$\alpha$-Terpineol & 1188 & 2.7 & - & 0.5 & - & 11.0 \\
\hline$\gamma$-Terpineol & 1199 & 0.9 & - & - & - & 3.0 \\
\hline Isobornyl acetate & 1285 & 2.0 & - & 2.6 & - & - \\
\hline$\delta$-Elemene & 1338 & - & - & - & 0.1 & - \\
\hline$\alpha$-Longipinene & 1352 & & & & - & 0.1 \\
\hline
\end{tabular}

\begin{tabular}{lcccccc}
\hline$\beta$-Patchoulene & 1381 & 0.4 & - & - & 4.9 & - \\
$\beta$-Elemene & 1390 & 0.1 & - & - & 0.9 & - \\
Cycloseychellene & 1407 & - & - & - & 0.6 & - \\
Longifolene & 1408 & - & - & - & - & 0.3 \\
E-Caryophyllene & 1419 & 0.6 & - & 0.6 & 2.2 & - \\
$\alpha$-Guaiene & 1439 & 1.0 & - & - & 15.0 & - \\
Seychellene & 1446 & 0.8 & - & - & 8.4 & - \\
$\alpha$-Humulene & 1454 & - & - & 0.1 & - & - \\
$\alpha$-Patchoulene & 1456 & 0.6 & - & - & 6.4 & - \\
Aciphyllene & 1501 & 0.2 & - & - & 2.9 & - \\
$\gamma$-Patchoulene & 1502 & 0.3 & - & - & 4.1 & - \\
$\alpha$-Bulnesene & 1509 & 1.3 & - & - & 20.8 & - \\
Nootkatene & 1518 & - & - & - & 0.2 & - \\
7-epi- $\alpha-S e l i n e n e$ & 1522 & - & - & - & 0.2 & - \\
Norpatchoulenol & 1555 & - & - & - & 0.4 & - \\
Caryophyllene oxide & 1583 & 0.2 & - & 0.3 & - & - \\
Pogostol & 1653 & - & - & - & 1.3 & - \\
Patchouli alcohol & 1658 & 1.7 & - & - & 31.6 & - \\
Cyperotundone & 1695 & - & - & - & 0.2 & - \\
\hline
\end{tabular}

*KI $=$ Kovats gas chromatographic retention indices of the peaks on the HP5MS column

The blended oil caused a significant increase of blood pressure. Since blood pressure is determined by the activity of the sympathetic branch of the ANS, an increase of blood pressure shows an increase in sympathetic tone, i.e., an increase of autonomic arousal (Andreassi, 2000; Heuberger et al., 2006; Hongratanaworakit et al., 2005; Hongratanaworakit et al., 2007; Hongratanaworakit, 2009; Hongratanaworakit, 2010; Hugdahl, 1995; Sayorwan et al., 2013).

In addition, PR of subjects significantly increased after sniffing the blended oil. Since pulse rate is mainly controlled by the ANS, the increase of pulse rate after sniffing of blended oil is likely to show a decrease in vagal tone, i.e., an increase of ANS arousal. The change in the cardiovascular parameter, i.e., pulse rate, was consistent with an increase of peripheral autonomic nervous activity (Andreassi, 2000; Heuberger et al., 2006; Hongratanaworakit et al., 2005; Hongratanaworakit et al., 2007; Hongratanaworakit, 2009; Hongratanaworakit, 2010; Hugdahl, 1995; Sayorwan et al., 2013). Inhalation of blended oil increased the level of arousal of the ANS, i.e. increases insystolic and diastolic blood pressure, mean arterial blood pressure, and pulse rate. Moreover, inhalation of blended oil led to activation at the behavioral level, i.e. subjects feel more alert, more vigorous, and more cheerful than before the administration of the oil. This finding points towards an increase of arousal in terms of selfevaluation(Andreassi, 2000; Heuberger et al., 2006; Hongratanaworakit et al., 2005; Hongratanaworakit et al., 2007; Hongratanaworakit, 2009; Hongratanaworakit, 2010; Hugdahl, 1995; Sayorwan et al., 2013). Thus, the effects of blended oil by inhalation may be characterized by the concept of stimulating/activating effects, which has also been described for rosemary oil (Hongratanaworakit, 2009; Sayorwan et al., 2013), kaffir lime oil (Hongratanaworakit et al., 2007), sandalwood oil(Heuberger et al., 2006), jasmine oil (Hongratanaworakit, 2010), and sweet orange oil (Hongratanaworakit et al., 2005). In addition, our findings clearly support previous studies indicating the stimulating effect of rosemary oil, patchouli oil, pine oil and 
the main chemical components in these oils (Albert and Steven, 1996; Atsumi and Tonosaki, 2007; Heuberger et al., 2000; Kovar et al., 1987; Lis-Balchin, 2006; Pujiarti et al., 2012; Steinmetz et al., 1987). Although our findings agree with other reports, it is important to assess further biochemical measures (e.g., noradrenaline), as these would further confirm the presence of stimulating/activating effects.

Blended oil mainly contained eucalyptus and rosemary oils. These essential oils contain abundant oxides and monoterpenes, and havethe main action of stimulating the nervous system under sympathetic control, leading to increase in alertness and vigor (Lis-Balchin, 2006; Sanders et al., 2002). Steinmetz et al.(1987)described the effect of 1,8-cineole and camphor, two of the constituents of blended oil, on the central nervous system. Their results showed both compounds stimulated the activity of the central nervous system, respiration, and circulation. Moreover, 1,8-cineole, the main component of blended oil, increased respiration rate after administration of the substance (Heuberger et al., 2008). Furthermore, pepper oil, estragon oil and grapefruit oil showed a stimulating effect on the sympathetic activity. These essential oils consist of some component, i.e. $\alpha$-pinene and $\beta$ pinene, which are possible that these components mediate the stimulating effect on the sympathetic activity (Hongratanaworakit, 2009; Lis-Balchin, 2006). Therefore, it is possible that these components such as 1,8-cineole, camphor or $\alpha$-pinene mediate the stimulating effect of the blended oil on the nervous system (Hongratanaworakit, 2009; Hugdahl, 1995).

\section{CONCLUSIONS}

In conclusion, our investigation demonstrates the stimulating effect of the blended oil. The blended oil showed significant increases of systolic blood pressure, diastolic blood pressure, mean arterial blood pressure and pulse rate which indicated an increase of autonomic arousal. In addition, inhalation of the blended oil led to activation at the behavioral level, i.e. subjects feel more alert, more vigorous, and more cheerful than before the administration of the oil. This finding points towards an increase of arousal in terms of self-evaluation. Thus, the effects of blended oil by inhalation may be characterized by the concept of stimulating/activating effects

\section{ACKNOWLEDGEMENTS} in the study.

The authors were grateful to the people who participated

Financial support and sponsorship: This work was supported by grants from Srinakharinwirot University (11/2014).

Conflict of Interests: There are no conflicts of interest.

\section{REFERENCES}

Adams RP. 2004. Identification of essential oil components by gas chromatography /quadrupole mass spectroscopy. Allured Publishing Corporation, Carol Stream, Illinois, USA.
Albert YL, Steven F. 1996. Encyclopedia of common natural ingredient 2nd ed. John Wiley \& Sons Inc., New York, United States.

Andreassi JL. 2000. Psychophysiology: human behavior \& physiological response. Lawrence Erlbaum Associated, London, UK.

Atsumi T, Tonosaki K. Smelling lavender and rosemary increase free radical scavenging activity and decrease cortisol level in saliva. Psychiat Res, 2007; 150: 89-96.

Bensafi M, Rouby C, Farget V, Bertrand B, Vigouroux M, Holley A. Autonomic nervous system responses to odors: the role of pleasantness and arousal. Chem Senses, 2000; 27: 703-709.

Chang SY. Effects of aroma hand massage on pain, state anxiety and depression in hospice patients with terminal cancer. TaehanKanhoHakhoe Chi, 2008; 38: 493-502.

Cooksley V. 1996. Aromatherapy: a lifetime guide to healing with essential oils. Prentice Hall, New Jersey, United States.

Damain P, Damain K. 1995. Aromatherapy: scent and psyche: using essential oils for physical and emotional well-being. Healing Arts Press, Vermont, United States.

Diego MA, Jones NA, Field T, Hernandez-Reif M, Schanberg S, Kuhn C. Aromatherapy positively affects mood, EEG patterns of alertness and math computations. Int J Neurosci, 1998; 96: 217-224.

Han SH, Hur MH, Buckle J, Choi J, Lee MS. Effect of aromatherapy on symptoms of dysmenorrheal in college students: A randomized placebo-controlled clinical trial. J Altern Complement Med, 2006; 12: 535-541.

Heuberger E, Hongratanaworakit T, Boehm C, Weber R, Buchbauer G. Effects of chiral fragrances on human autonomic nervous system parameters and self-evaluation. Chem Senses, 2000; 26: 281-292.

Heuberger E, Hongratanaworakit T, Buchbauer G. East Indian sandalwood oil and alpha-santalol odor increase physiological and selfrated arousal in humans. Planta Med, 2006; 72: 792-800.

Heuberger E, Ilmberger J, Hartter E, Buchbauer G. Physiological and behavioral effects of 1,8-cineole and (+/-)-linalool: A comparison of inhalation and massage aromatherapy. Nat Prod Commun, 2008; 3: 1103-1110.

Hongratanaworakit T, Buchbauer G. Human behavioral and physiological reactions to inhalation of sweet orange oil. ActaHort, 2005; 679: 75-81.

Hongratanaworakit T, Buchbauer G. Chemical composition and stimulating effect of Citrus hystrix oil on humans. FlavourFragr J, 2007; 22: 443-449.

Hongratanaworakit T. Simultaneous aromatherapy massage with rosemary on humans. Sci Pharm, 2009; 77: 375-387.

Hongratanaworakit T. Stimulating effect of aromatherapy massage with jasmine oil. Nat Prod Commun, 2010; 5:157-161.

Hudson CM. 1999. Aromatherapy massage. Dorling Kindersley Limited, London, UK.

Hugdahl K. 1995. Psychophysiology: the mind-body perspective. University Press, England.

Imura $\mathrm{M}$, Misao $\mathrm{H}$, Ushijima $\mathrm{H}$. The psychological effects of aromatherapy-massage in healthy postpartum mothers. J Midwifery Womens Health, 2006; 51: e21-e27.

Inoue $\mathrm{N}$, Kuroda $\mathrm{K}$, Sugimoto A, Kakuda $\mathrm{T}$, Fushiki $\mathrm{T}$. Different autonomic nervous responses according to preference for the odor of jasmine tea. BiosciBiotechnolBiochem, 2003; 67: 1206-1214.

Kovar KA, Gropper B, Friess D, Ammon HTP. Blood level of 1,8-cineole and locomotor activity of mice after inhalation and oral administration of rosemary oil. Planta Med, 1987; 53: 315-319.

Lakusic D, Ristic M, Slavkovska V, Lakusic B. Seasonal variations in the composition of the essential oils of rosemary (Rosmarinus officinalis, Lamiaceae) Nat Prod Commun, 2013; 8: 131-134.

Lis-Balchin M. 2006. Aromatherapy science: a guide for healthcare professionals. Pharmaceutical Press, London, England.

Moss M, Cook J, Wesnes K, Duckett P. Aromas of rosemary and lavender essential oils differentially affect cognition and mood in healthy adults. Int J Neurosci, 2003; 113: 15-38.

Pujiarti R, Ohtani Y, Widowati TB, Kasmudjo W, Herath NK, Wang CN. Effect of Melaleuca leucadendron, Canangaodorata and 
Pogostemoncablin oil odors on human physiological responses. Wood Research Journal, 2012; 3: 100-105.

Rutledge DN, Jones CJ. Effects of topical essential oil on exercise volume after a 12-week exercise program for women with fibromyalgia: a pilot study. J Altern Complement Med, 2007; 13: 10991106.

Sanders C, Diego M, Fernandez M, Field T, Hermandez-Reif $\mathrm{M}$, Roca A. EEG asymmetry response to lavender and rosemary aromas in adults and infants. Int J Neurosci, 2002; 112: 1305-1320.

Sayowan W, Siripornpanich V, Piriyapunyaporn T, Hongratanaworakit T, Kotchabhakdi N, Ruangrungsi N. The effects of lavender oil inhalation on emotional states, autonomic nervous system and brain electrical activity. J Med Assoc Thai, 2012; 95(4): 589-606.

Sayorwan W, Ruangrungsi N, Piriyapunyporn $\mathrm{T}$, Hongratanaworakit T, Kotchabhakdi N, Siripornpanich V. Effects of inhaled rosemary oil on subjective feelings and activities of the nervous system. Sci Pharm, 2013; 81: 531-542.
Steinmetz MD, Vial M, Millet Y. Actions of essential oils of rosemary and certain of its constituents (eucalyptol and camphor) on the cerebral cortex of the rat in vitro. J ToxicolClinExp, 1987; 7: 259-271.

Taorang B, Rawdlaurh P, Pumtong S, Hongratanaworakit T. Formulation of essential oil preparation for relaxation. J Thai TraditAltern Med, 2009; 7(supplement), 67.

Wildwood C. 2000. Aroma remedies. Collins \& Brown Limited, London, UK.

\section{How to cite this article:}

Hongratanaworakit T, Srimuang $\mathrm{P}$, Wichanpreechar A, Akarapattaranithi T, Taorang B, Rawdlaurh P., Chemical Composition and Effects of Blended Essential Oil on Humans. J App Pharm Sci, 2017; 7 (01): 165-170. 